\title{
Structural behavior of cylindrical polystyrene-b-poly(ethylene-butylene)-b- polystyrene (SEBS) triblock copolymer containing MWCNTs: On the influence of nanoparticle surface modification
}

Noushin Hasanabadi ${ }^{1}$, Hossein Nazockdast ${ }^{1 *}$, Bernadetta Gajewska ${ }^{2}$, Sandor Balog $^{2}$, Ilja Gunkel ${ }^{2}$, Nico Bruns ${ }^{2}$, Marco Lattuada ${ }^{2 *}$

\author{
1) Noushin Hasanabadi, Prof. Hossein.Nazockdast \\ Department of Polymer Engineering, Amirkabir University of Technology, 15875-4413 \\ Tehran, Iran \\ E-mail: nazdast@aut.ac.ir \\ ${ }^{2)}$ Bernadetta Gajewska, Dr. Sandor Balog, Dr. Ilja Gunkel, Prof. Nico Bruns \\ Adolphe Merkle Institute, University of Fribourg, Chemin des Verdiers 4, CH-1700 Fribourg, \\ Switzerland \\ 3) Prof. Marco Lattuada \\ Department of Chemistry, University of Fribourg, Chemin du Musée 9, CH-1700 Fribourg, \\ Switzerland
}

E-mail: Marco.lattuada@unifr.ch

\begin{abstract}
In this work, the influence of carbon nanotubes (CNTs) on the self-assembly of nanocomposite materials made of cylinder-forming polystyrene-b-poly(ethylene-butylene)-b-polystyrene (SEBS) is studied. CNTs are modified with polystyrene (PS) brushes by surface-initiated atom transfer radical polymerization to facilitate both their dispersion and the orientation of neighboring PS domains of the block copolymer (BCP) along modified CNT-PS. Dynamic rheology is utilized to probe the viscoelastic and thermal response of the nanoscopic structure of BCP nanocomposites. The results indicate that non-modified CNTs increase the BCP microphase separation temperature because of BCP segmental confinement in the existing 3D network formed between CNTs, while the opposite holds for the samples filled with modified CNT-PS. This is explained by severely retarded segmental motion of the matrix chains due to their preferential interactions with the PS chains of the CNT-PS. Moreover, transient viscoelastic analysis reveals that modified CNT-PS have a more pronounced effect on flow-
\end{abstract}


induced BCP structural orientation with much lower structural recovery rate. It is demonstrated that dynamic-mechanical thermal analysis can provide valuable insights in understanding the role of CNT incorporation on the microstructure of BCP nanocomposite samples. Accordingly, the presence of CNT has a significant promoting effect on microstructural development, comparable to that of annealing.

\section{TOC image}

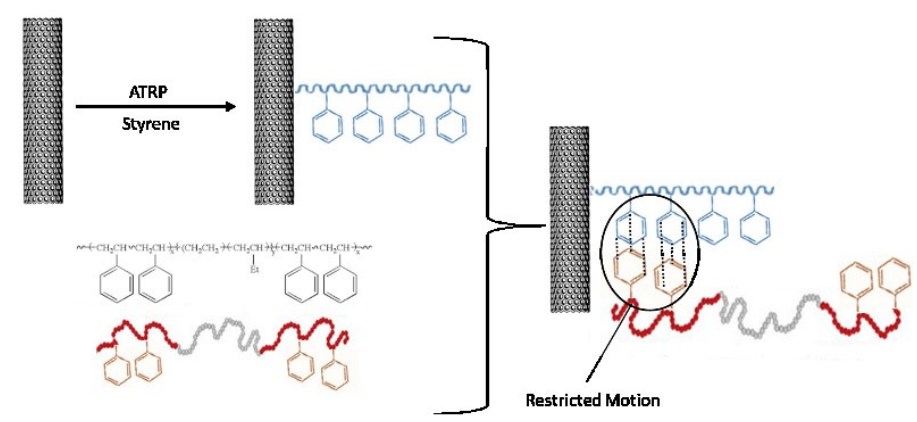




\section{Introduction}

Block copolymers (BCPs) represent a subject of broad current research due to their ability to generate nanoscale ordered structures (one of the components of the BCP dispersed in a matrix of the other component), such as spheres, cylinders, gyroids and lamella. ${ }^{[1]}$ This kind of molecular self-assembly is the direct consequence of thermodynamic incompatibility of the chemically-linked blocks of the block copolymer. ${ }^{[2-4]}$ The complex microstructure of BCP materials does not only render them interesting for purely academic purposes, but also because of their wide range of applications such as photolithography, medicine, electronics and gas separation. ${ }^{[5-8]}$ In order to further improve their properties, various studies have investigated the incorporation of nanoparticles into block copolymers as a highly promising method to tailor the material's properties and enhance their mechanical, electrical and thermal properties. ${ }^{[9-17]}$ Carbon nanotubes (CNTs) are very promising nanomaterials due to the combination of their superlative thermal, mechanical and electrical properties. ${ }^{[18-21]} \mathrm{CNTs}$ tend to agglomerate and form strongly entangled bundles, driven by van der Waals forces. This renders their dispersion into polymer matrices challenging and inhibits the translation of their nanophysical properties to nanocomposites. Therefore, the challenging issue in multifunctional polymer nanocomposites based on CNTs is to achieve a high degree of CNT dispersion in the host polymer. ${ }^{[19]}$ One approach to address this issue is to adjust the thermodynamic interaction between the $\mathrm{BCP}$ and $\mathrm{CNTs}$ either by using surfactants or by grafting a polymer brush on the surface of the CNTs, which is chemically identical to one block of the BCP. Peponi et al. demonstrated how dodecanethiol (DT) could be used as an efficient surfactant to sequester octadecylamine functionalized single-walled carbon nanotubes (ODA-SWCNTs) into the polystyrene (PS) domains of self-assembled polystyrene- $b$-polyisoprene- $b$-polystyrene (SIS) ${ }^{[22,23]}$ Moreover, the surfactant assisted the confinement of CNT into the PS phase of the materials as confirmed by electrostatic force microscopy (EFM) and by an increase of the glass 
transition temperature of PS due to the better interaction between modified CNT and the PS phase.

Calisi et al. reported two methodologies to investigate the factors affecting the dispersion of CNTs within SEBS. The first depended on the sonication time while the second relied on the use of alkyl-functionalized MWCNTs (f-MWCNTs). TEM micrographs revealed that prolonged sonication time induced an extensive CNT degradation while CNT functionalization appeared to be an effective method in preparing nanocomposites with high dispersion and negligible degradation of CNTs. ${ }^{[22]}$

SEBS-functionalized multi-walled carbon nanotubes (MWCNTs) could be prepared by covalently attachment of azide-containing SEBS with alkyne-decorated MWCNTs via click chemistry. The covalent functionalization of MWCNTs with SEBS leads to better dispersion of the CNTs in the SEBS polymer matrix. Therefore, enhanced mechanical properties, higher dielectric constant and greater thermal stability could be achieved. ${ }^{[25]}$

Mondragon et al. described the synergistic effect of using PS-grafted MWCNT and dodecanethiol as a surfactant in the dispersion of MWCNT in SIS block copolymers. This synergistic effect is due to the interaction between DT molecules and grafted PS chains on the CNTs, which improved the dispersion of PS-CNT in solution. ${ }^{[26]}$ Ilcikova et al. also studied the effect of incorporation of PS-CNT in SIS block copolymer. Dynamic mechanical analysis was utilized to demonstrate the improved interaction of modified MWCNT compared to neat MWCNT, leading improved elastic properties of the composite materials. ${ }^{[15]}$ Based on the " grafting from" approach to modify the surface of CNTs, they focused on using in situ grafting of a block copolymer to prepare nanocomposite samples with superior CNTs dispersion and distribution. These nanocomposite samples were prepared by the in situ grafting of CNTs with poly n-butyl acrylate-poly methyl methacrylate (PBA-b-PMMA) diblock copolymer during the synthesis of the linear triblock copolymer poly(methyl methacrylate)-b-poly(n-butyl acrylate)b-poly(methyl methacrylate) (PMMA-b-PBA-b-PMMA). ${ }^{[27,28]}$ These examples show that 
significant progress has been made to modify interfacial interactions between $\mathrm{CNT}$ and $\mathrm{BCP}$ in order to promote the dispersion of the nanotubes, which is required for enhancing prescribed properties. Although the above-mentioned progress in the selective location of CNT in BCP is of great value, some aspects of these materials, including the effect of CNTs and their modification on the $\mathrm{BCP}$ microphase separation temperature and on the kinetics of microphase separation, have not been fully understood.

Given the importance of understanding the kinetics of microphase separation for the processing and for the final properties of these composite materials, in the present work we investigated the effect of CNT on the microstructural evolution of commercially available SEBS block copolymers by employing a combination of rheological and thermal analysis. To further clarify the role of surface modification of CNTs on the composite properties, PS brushes were grown on the surface of CNTs by atom transfer radical polymerization (ATRP). The properties of composite materials made with modified CNTs were compared to those of prepared with neat CNTs.

\section{Experimental Section}

\subsection{Materials}

A commercial SEBS block copolymer with $30 \mathrm{wt} \%$ PS content (SEBS G1652; solution viscosity at $25{ }^{\circ} \mathrm{C}$ and $25 \mathrm{wt} \%$ in toluene: $1800 \mathrm{cps}$ ) was kindly supplied by Kraton Corporation. Multi-walled carbon nanotubes, hereafter abbreviated as CNTs, were purchased from Nanocyl under the trade name Nanocyl NC 7000, with an average outer diameter of $9.5 \mathrm{~nm}$ and average length up to $1.5 \mu \mathrm{m}$. Isoamyl nitrite, diethylether, triethylamine $\left(\mathrm{Et}_{3} \mathrm{~N}\right)$, styrene, anisole, copper(I) bromide (CuBr), 2-(4-aminophenyl)ethanol, 2-bromopropionyl bromide, methyl 2bromopropionate, $N, N, N$ ', $N$ ', $N$ ' '-pentamethyl diethylenetriamine (PMDETA) and membranes Durapore PVDF pore size $0.45 \mu \mathrm{m}$ (Milipore HVLP4700) were purchased from Sigma Aldrich. Acetone and dimethylformamide (DMF) were obtained from Honeywell. 
Styrene stabilizers were removed by passing the monomer through a plug of dry, activated basic alumina prior to use. All other chemicals and solvents were used without any further purification unless otherwise stated.

\subsection{Synthesis of CNT-OH}

Modification of CNT with PS chains was carried out according to the approach of Ilcikova et

al. $^{[15]}$ The first step requires the introduction of hydroxyl groups on the surface of pristine CNTs. For this purpose, $2.000 \mathrm{~g} \mathrm{CNT}$ and $11.000 \mathrm{~g}(0.08 \mathrm{~mol})$ 2-(4-aminophenyl)ethanol were loaded in a $250 \mathrm{~mL}$ three-neck round-bottom flask equipped with a condenser. The flask was evacuated and backfilled with nitrogen five times. $44.5 \mathrm{~mL}$ (38.8 mg, $0.33 \mathrm{~mol})$ degassed isoamyl nitrite were added. Subsequently, the flask was transferred to a pre-heated oil bath $\left(60^{\circ} \mathrm{C}\right)$ and the reaction mixture was stirred for $1 \mathrm{~h} .10 \mathrm{~mL}$ of degassed tetrahydrofuran was added and the reaction mixture was sonicated for $30 \mathrm{~min}$, followed by stirring at $60{ }^{\circ} \mathrm{C}$ for additional $2 \mathrm{~h}$. The suspension was then filtered over a $0.45 \mu \mathrm{m}$ PTFE membrane filter and washed with acetone. Then, the filter cake was dispersed in $200 \mathrm{~mL} \mathrm{DMF}$, sonicated for $10 \mathrm{~min}$ at room temperature, filtered and washed with acetone. This procedure was repeated three times to remove all the unreacted organics until the filtrate became clear. Finally, the product was washed with $100 \mathrm{~mL}$ diethyl ether and dried in a vacuum oven at $60^{\circ} \mathrm{C}$ overnight.

\subsection{Modification of CNT-OH with 2-bromopropionyl Groups (CNT-Br)}

$2.000 \mathrm{~g}$ CNT-OH was put into a $100 \mathrm{~mL}$ three-neck round-bottom flask. The reaction flask was evacuated and backfilled with nitrogen three times. $400 \mathrm{~mL}$ dry THF was added using a syringe. After ultrasonication for $30 \mathrm{~min}$, the flask containing the mixture was transferred to a $0{ }^{\circ} \mathrm{C}$ bath and $1 \mathrm{~mL}$ triethylamine was added, followed by a dropwise addition of $1.5 \mathrm{~mL} 2$ - 
bromopropionyl bromide. Stirring was continued overnight at room temperature. Then, the reaction mixture was refluxed at $70{ }^{\circ} \mathrm{C}$ for $1 \mathrm{~h}$. The solid was separated from the mixture by filtration using a $0.45 \mu \mathrm{m}$ PTFE membrane and washed with acetone. The filter cake was then re-dispersed in $200 \mathrm{~mL}$ DMF, shortly sonicated $(1 \mathrm{~min})$, filtered and washed with acetone. The dispersing, filtering and washing steps were repeated three times to assure removal of all the unreacted organic reagents. After washing the filter cake with $100 \mathrm{~mL}$ diethylether, the resulting black solid was dried under vacuum at $60{ }^{\circ} \mathrm{C}$.

\subsection{Synthesis of Polystyrene-modified CNT (CNT-PS)}

ATRP was performed in a $100 \mathrm{~mL}$ Schlenk flask. $400 \mathrm{mg}$ of the CNT-Br was placed into the flask, which was evacuated and backfilled with nitrogen gas three times and then filled with nitrogen. $0.18 \mathrm{~mL}$ (0.14 mg, $0.86 \mathrm{mmol})$ PMDETA dissolved in $2 \mathrm{~mL}$ anisole, $26.9 \mathrm{~mL}$ (24.37 $\mathrm{mg}, 0.23 \mathrm{~mol})$ styrene and $0.19 \mathrm{~mL}(0.28 \mathrm{mg}, 1.7 \mathrm{mmol})$ methyl 2-bromopropionate dissolved in $14.1 \mathrm{~mL}$ anisole were separately purged with nitrogen and transferred into the Schlenk flask under nitrogen flow. The mixture was sonicated for $10 \mathrm{~min}$ and then was degassed by four freeze-pump-thaw cycles. $120.5 \mathrm{mg}(0.84 \mathrm{mmol}) \mathrm{CuBr}$ was added to the frozen reaction mixture under nitrogen flow and one more freeze-pump-thaw cycle was performed and flask was left under nitrogen. The final molar ratio of PMDETA/styrene/methyl-2-bromopropoinate/ $\mathrm{CuBr}$ was $1 / 267 / 1.97 / 0.97$. Subsequently, the suspension was immersed in an oil bath at $80{ }^{\circ} \mathrm{C}$ while stirring continuously for $28.5 \mathrm{~h}$. The polymerization was stopped by opening the flask to air and dissolving the mixture in $50 \mathrm{~mL}$ non-deoxygenated acetone. PS chains that were not grafted to the surface of CNTs were separated from the CNT-PS conjugates by centrifugation $(3000 \mathrm{~g})$ at room temperature for $30 \mathrm{~min}$. CNT-PS was re-dispersed in acetone, sonicated and centrifuged again. This procedure was repeated three times and the purified CNT-PS was dried overnight in a vacuum oven at $60^{\circ} \mathrm{C}$. 
The CNT-PS were analyzed using energy-dispersive X-ray spectroscopy (EDX) incorporated into an SEM (TESCAN Mira 3 LM Field Emission). The quantitative results of the EDX analysis are reported in Table 1. In addition to carbon and oxygen, EDX confirmed the presence of bromine, present at the end of the polymer chains (or as an unreacted initiator), and copper, used as catalyst for the ATRP.

Table 1. Quantitative results of the EDX analysis performed on the CNT-PS samples. For each element, in addition to the individual peak intensity (count rate), the weight percentage as well as the atomic percentage of each elemt is reported.

\begin{tabular}{|c|c|c|c|}
\hline Element & Peak Intensity [a.u.] & Atomic \% & Weight \% \\
\hline Carbon & 939.0 & 92.01 & 86.15 \\
\hline Oxygen & 44.3 & 7.17 & 8.94 \\
\hline Copper & 4.9 & 0.15 & 0.73 \\
\hline Bromine & 152.3 & 0.67 & 4.19 \\
\hline
\end{tabular}

\subsection{Preparation of Composites}

Nanocomposite samples for rheological analysis containing either $0.25 \mathrm{wt} \% \mathrm{CNT}$ or $0.75 \mathrm{wt} \%$ CNT-PS (corresponding to $0.25 \mathrm{wt} \%$ neat CNT) were prepared by melt compounding the polymer and the CNTs (as solids) in a laboratory internal mixer, $60 \mathrm{~cm}^{3}$, equipped with a banbury-type rotor design (Brabender Plasticorder W5) at $220{ }^{\circ} \mathrm{C}$ and rotor speed of $80 \mathrm{rpm}$ for a total mixing time of $15 \mathrm{~min}$. Samples were removed from the mixer and characterized without any further annealing or processing. Note that the difference in the amount of CNT and CNTPS added to the system is due to the amount of polymer (about $68 \%$ ) bound to the CNTs surface. In this manner, the same amount of CNT is present in both types of composite materials. 
To prepare samples for AFM imaging, SEBS BCPs were first dissolved in chloroform at a fixed polymer concentration of $20 \mathrm{mg} / \mathrm{ml}$. The required amounts of CNT or of CNT-PS were then added to the SEBS BCP solution to obtain nanocomposite samples with the desired composition, followed by sonicating the mixture for $2 \mathrm{~h}$ to ensure homogenous dispersion. Thin films were prepared by spin coating the nanocomposite solution at $2500 \mathrm{rpm}$ for $20 \mathrm{~s}$ onto silicon wafer pieces at ambient conditions, and subsequently annealed in nitrogen atmosphere at $180{ }^{\circ} \mathrm{C}$ for $1 \mathrm{~h}$.

\subsection{Characterization}

The average molecular weight and molecular weight distribution of PS chains grown from the free initiator were determined by gel permeation chromatography (GPC) using an Agilent 1200 series HPLC system equipped with an Agilent PLgel mixed guard column (particle size $=5$ $\mu \mathrm{m})$, two Agilent PLgel mixed-D columns $(\mathrm{ID}=7.5 \mathrm{~mm}, \mathrm{~L}=300 \mathrm{~mm}$, particle size $=5 \mu \mathrm{m})$ and an Optilab REX interferometric refractometer detector. THF was used as an eluent at a flow

rate of $1 \mathrm{~mL} \mathrm{~min}^{-1}$. The GPC was calibrated with narrow molecular weight PS standards (2340 to $275300 \mathrm{~g} \mathrm{~mol}^{-1}$ ). Data analysis was carried out with the Astra software (Wyatt Technology Corp).

Thermogravimetric analysis (TGA) was conducted on a Mettler Toledo TGA/DGSC 1 Star $^{\mathrm{e}}$ system under nitrogen atmosphere in a temperature range from room temperature $\left(25^{\circ} \mathrm{C}\right)$ to $600{ }^{\circ} \mathrm{C}$ at the heating rate of $10^{\circ} \mathrm{C} \mathrm{min}^{-1}$.

The melt state viscoelastic behavior of the samples was studied using a rheometric mechanical spectrometer (Paar Physica UDS 200). All measurements were carried out utilizing parallel plate geometry with a diameter of $25 \mathrm{~mm}$ and a sample gap of $1 \mathrm{~mm}$ under nitrogen atmosphere to prevent any thermal degradation during the measurements. A small amount of either the neat $\mathrm{BCP}$ or the composite material was squeezed between the plates. The linear viscoelastic 
properties of samples were examined by using frequency sweep experiments in small oscillatory shear deformation over frequencies ranging from 0.05 to $1000 \mathrm{~s}^{-1}$ with a fixed strain of $1 \%$. Isochronal dynamic temperature sweep tests were carried out to study the microphase separation behavior of samples by monitoring storage modulus at a fixed frequency of $1 \mathrm{~s}^{-1}$, strain of $1 \%$ and a uniform cooling rate of $1{ }^{\circ} \mathrm{C} \min ^{-1}$ under nitrogen atmosphere. To study the structural reformation kinetics of the samples, a transient rheological experiment followed by a time sweep experiment was performed according to the following procedure: (i) a steady shear deformation with a constant shear rate of $1 \mathrm{~s}^{-1}$ was exerted on molten testing samples for $500 \mathrm{~s}$ and (ii) small amplitude oscillatory shear (SAOC) time sweep experiment $\left(\omega=1 \mathrm{~s}^{-1}\right.$, strain=1) was performed immediately after the shear flow had ceased in order to evaluate the extent of structural recovery.

Dynamic mechanical thermal analysis (DMTA) was recorded by a Diamond DMA Perkin Elmer instrument in tensile mode on samples of $20 \times 11 \times 1 \mathrm{~mm}^{3}$ cut from compression molded sheets. Thermograms were measured at frequency of $1 \mathrm{~Hz}$ and a heating rate of $5{ }^{\circ} \mathrm{C} \mathrm{min}-1$ over temperature range from -100 to $140{ }^{\circ} \mathrm{C}$.

The morphology of the prepared samples was investigated by small-angle X-ray scattering (SAXS). SAXS measurements were performed with a NanoMax-IQ camera (Rigaku Innovative Technologies, Auburn Hills, MI USA) at room temperature. 2-dimensional SAXS patterns were radially averaged yielding 1D SAXS curves of I(q), $q$ being the scattering vector $\left(q=4 \pi \lambda^{-1}\right.$ $\sin (\theta / 2)$, where $\theta$ and $\lambda=0.1524 \mathrm{~nm}$ are scattering angle and photon wavelength, respectively). The surface structure of SEBS BCP films was imaged by atomic force microscopy (AFM) on a JPK Nanowizard II with a JPK Advanced SPM control station. AFM imaging was performed in tapping mode using rectangular Al-coated silicon cantilevers (NanoAndMore GmbH, $k=40$ $\mathrm{N} / \mathrm{m}, f=300 \mathrm{kHz}$ ) integrated with silicon tips (tip radius $<10 \mathrm{~nm}$ ). 


\section{Results and Discussion}

The objective of this work is to investigate the role CNT on the microphase separation properties of SEBS block copolymers. In addition to neat CNT, CNTs were modified by grafting PS chains via surface initiated ATRP, to study the effect of thermodynamic interactions between CNTs and the SEBS matrix on the microstructure of the composite materials.

\subsection{Modification of CNT Surface}

In order to grow PS chains from the surface of the CNTs using ATPR, their surface needs to be functionalized with an ATRP initiator. This was accomplished by first grafting hydroxyl groups on their surface (through a reaction with 2-(4-aminophenyl) ethanol and isoamyl nitrite). Subsequently, esterification with 2-bromopropionyl bromide was used to anchor the ATRP initiator to the CNTs, before starting the polymerization of styrene. Simultaneously to the grafting-from polymerization, free non-grafted PS chains were polymerized from sacrificial initiator in order to confirm that the reaction conditions allowed for a well-controlled ATRP. These chains had a number average molecular weight and dispersity of $\mathrm{M}_{\mathrm{n}}=1770 \mathrm{~g} \mathrm{~mol}^{-1}$ and $\mathrm{D}=1.06$, respectively. Thus, it can be assumed that the chains grafted from the surface are likely to have a relatively low molecular weight and a narrow molecular weight distribution.

In order to quantify the content of grafted PS on the surface of CNT-PS, thermogravimetric measurements (TGA) were carried out. As it can be observed in Figure 1, pristine CNTs hardly decompose below $500{ }^{\circ} \mathrm{C}$. Their major mass loss starts at $500{ }^{\circ} \mathrm{C}$. The hydroxylated CNTs and CNT-Br show a smooth weight loss starting at $150{ }^{\circ} \mathrm{C}$. This can be related to the degradation of hydroxyl groups and initiator molecules that are covalently attached on the surface of the CNTs. In contrast, CNT-PS exhibits a significant weight loss starting at $300{ }^{\circ} \mathrm{C}$ and proceeding 
up to $500{ }^{\circ} \mathrm{C}$. It corresponds to the degradation of PS chains. The temperature at which this happens is typical for the decomposition of PS. ${ }^{[29]}$ The difference between the weights of the CNT-Br and the CNT-PS weight at $600{ }^{\circ} \mathrm{C}$ corresponds to the PS-content of CNT-PS, which was approximately $68 \mathrm{wt} \%$. This means that the mass of polymer amounts to about $2 / 3$ of the overall mass of the CNT-PS. Note that at $600^{\circ} \mathrm{C}$ the difference in mass loss between the bare CNT, CNT-Br and CNT-OH is negligible, and was therefore used as a reference temperature.

Fig. 1

\subsection{Structure of CNT/SEBS Nanocomposite in Thin Films}

In order to better understand the behavior of CNTs dispersed in SEBS BCPs, AFM imaging has been carried out. The difficulty of preparing samples for AFM imaging using melt compounding was circumvented via preparing thin nanocomposite films by means of spincoating from solutions containing both the polymer and the CNTs. In this manner, sufficiently flat samples could be prepared, suitable for AFM imaging. Three representative samples have been imaged, and are shown in Figure 2. The neat SEBS BCP (Figure 2a), shows a structure consistent with cylindrical microdomains oriented parallel to substrate surface. Note that while this morphology is consistent with the SAXS results obtained for neat SEBS in the bulk (see below), the surface pattern in Figure 2a was only obtained after annealing neat SEBS BCP films at $180^{\circ} \mathrm{C}$ for $1 \mathrm{~h}$. The AFM image of an SEBS BCP film containing non-modified CNTs (Figure $2 \mathrm{~b}$ ) indicates no preferential arrangement of the CNTs, which appear to be positioned across different domains. In SEBS BCP films containing functionalized CNTs (Figure 2c), however, the nanotubes were found to be located exclusively in the PS domains, which appear to align along the nanotubes in order to accommodate the nanotubes. This suggests that that surface modification of CNT significantly helps compatibilizing CNT with the BCP matrix. 
Fig. 2

\subsection{Morphology of CNT/SEBS BCP Nanocomposites in the Bulk}

SAXS was used to obtain structural information on the CNT/BCP nanocomposite samples. Their SAXS profiles are shown in Figure 3. Bragg peaks appear at relative q-positions of $1, \sqrt{ }$ 3 and $\sqrt{7}$, indicating all samples exhibit a morphology of hexagonally packed cylinders. ${ }^{[9,30,31]}$ The similar morphologies observed in CNT/SEBS nanocomposite and neat SEBS BCP samples implies that the presence of CNTs, both functionalized and non-functionalized, does not affect the morphology of the copolymer. Note that the peaks characteristic of the cylindrical morphology are slightly broader for the composite samples than for the neat BCP, which suggests that the presence of the CNTs leads to a deterioration of the packing of the cylindrical microdomains.

Distance between BCP styrenic cylindrical domains were determined using the values of first Bragg peak $\left(\mathrm{d}=2 \pi / \mathrm{q}^{*}\right)$. The values are $23.8,23.5$ and $24.3 \mathrm{~nm}$ for neat $\mathrm{BCP}$ and its nanocomposites containing pristine CNT and modified CNT-PS, respectively. The increased BCP domain spacing upon incorporation of modified CNT-PS is due to the great preferential interaction of CNT-PS and PS segments of the block copolymer while the decreased domain spacing with the presence of unmodified CNT is attributed to the sever confinement effect of nanoparticles. The above-mentioned reasons would be discussed in rheological section.

Fig. 3

\subsection{Melt State Rheological Behavior of CNT/SEBS Composites}


In order to investigate the effect of CNT incorporation into SEBS block copolymer materials, key viscoelastic properties of the neat block copolymer, of the copolymer with unmodified CNT and of the one with modified CNTs were measured in small amplitude oscillations as a function of frequency. From these experiments, the frequency dependence of the storage and loss moduli can be obtained. One should note that the mass fraction of PS-modified CNTs in the composite material is different from that of neat CNTs in order to account for the mass of grafted PS chains. The composites were prepared to have the same mass fraction of pure CNTs in the composite. Figure 4 shows the results of storage modulus, $\mathrm{G}^{\prime}$, as a function of frequency for neat SEBS BCP and its nanocomposites containing pristine CNT and modified CNT-PS measured at $220{ }^{\circ} \mathrm{C}$. The storage modulus for pure polymer melts is expected to scale as a power law of a frequency, with an exponent equal to two in the low frequency regime. As can be observed, the slope of storage modulus of neat SEBS at low frequency range exhibits a pronounced negative deviation from two, reported for most polymer melts (Rouse viscoelastic model). Such nonterminal behavior of storage modulus at low frequency $\left(\mathrm{G}^{\prime} \sim \omega^{0.21}\right)$ is the characteristic of $\mathrm{BCP}$ with cylindrical structure caused by the 3-D network structure formed by randomly oriented and interconnected cylindrical domains, whose interconnection is not completely destroyed even at $220^{\circ} \mathrm{C} .{ }^{[10]}$ It is worth noting that, according to the self-consistent field theory, SEBS G1652 BCP is expected to have a cylindrical microstructure due to the PS weight fraction of $30 \%$, which is in good agreement with the rheological results. ${ }^{[4]}$

The $0.25 \mathrm{wt} \% \mathrm{CNT}$ containing sample shows an even more pronounced low frequency nonterminal storage modulus behavior due to the strong three-dimensional network structure formed by CNT-CNT and/or CNT-matrix interactions. On the other hand, the nanocomposite sample containing $0.75 \mathrm{wt} \%$ modified CNT-PS exhibits a completely different rheological behavior than the other samples. The addition of modified CNT-PS enhances the storage modulus of $\mathrm{BCP}$ at low frequency range in comparison to neat $\mathrm{BCP}$ while it has a reverse effect 
at high frequencies. The observed rheological results can be clearly explained by the stronger and improved interaction between CNT-PS and PS segments of SEBS.

The lower storage modulus at high frequency of the modified CNT-PS filled sample in comparison to neat $\mathrm{BCP}$ is the result of a lower number of free $\mathrm{BCP}$ chains that do not have any particular interaction with modified CNT-PS nanoparticles and therefore experience shortrange mobility. Moreover, at the low weight percentage of modified CNT-PS $(0.75 \mathrm{wt} \%)$, it is likely that each CNT is encapsulated with BCP PS segments. As a result, the CNT-PS could not form a three-dimensional network structure and are well dispersed in the BCP matrix. Furthermore, the strong interaction between modified CNT-PS and PS segments imparts additional conformational constrains to the long-range segmental mobility. Hence, the enhanced storage modulus at low frequencies in comparison with neat $\mathrm{BCP}$ is due to the restriction of the terminal relaxation.

Fig. 4

Melt rheology is a powerful tool that can provide information on the microstructural evolution of BCPs as a function of temperature. Microphase separation temperature of BCP can be estimated through a distinct change in the slope of the storage modulus curve vs. temperature, as a result of microphase separation-induced elastic (solid-like) response. ${ }^{[32,33]}$ The results of temperature sweep experiment performed on neat SEBS and its nanocomposite samples are shown in Figure 5. The microphase separation temperature of SEBS and nanocomposite samples containing $0.25 \mathrm{wt} \% \mathrm{CNT}$ and $0.75 \mathrm{wt} \%$ modified CNT-PS were found to be 129.5 , 133.7 and $127.7^{\circ} \mathrm{C}$, respectively.

The microphase separation temperature of $\mathrm{BCP}$ is mainly governed by two opposing forces: enthalpic interactions and entropy. From the entropic point of view, the BCP is favored to be in a disordered state with maximum number of possible conformations. However, enthalpic interaction leads to microphase separation in order to minimize the unfavorable interactions 
between different blocks. As a consequence, the enhanced BCP microphase separation temperature caused by the presence of neat $\mathrm{CNT}$ is attributed to to the confinement of $\mathrm{BCP}$ chains between CNT particles. Such confinement leads to the stretching of polymer chains along nanotubes and reducing the number of chain conformations. Therefore, the enthalpic contribution begins to dominate over entropic contribution ${ }^{[34,35]}$ and microphase separation occurs at higher temperature.

On the other hand, the presence of modified CNT-PS reduces the BCP microphase separation temperature. Under these circumstances, the increased interfacial interaction between the PS grafts on the surface of the CNTs and the PS segments of the BCP restricts the segmental motion of PS and therefore the chain's ability to be arranged into separate domains and form a microphase separated structure.

Fig. 5

\subsection{Transient Flow Measurements}

Transient flow measurements probe the viscoelastic behavior of materials under shear. The viscoelastic behavior of BCPs is strongly influenced by the applied flow field. Shear deformation may affect the orientation of $\mathrm{BCP}$ domains. This behavior becomes more complex in the presence of anisotropic nanoparticles. Moreover, the structural reconstructing during rest upon cessation of flow is of great importance for the end application of the material. The time evolution of the nanocomposites BCP structure was evaluated by using transient rheological test. Figure 6a presents transient shear stress as a function of shearing time for neat $\mathrm{BCP}$ and nanocomposite samples, exposed to a constant shear rate of $1 \mathrm{~s}^{-1}$. The neat BCP exhibits a strong stress overshoot which quickly levels off to a steady-state value. This behavior is attributed to the BCP three-dimensional interconnected microstructure formed by the cylindrical domains, which is partially preserved even at a temperature of $220^{\circ} \mathrm{C}$. It can be observed that the presence 
of CNTs in the BCP results in stronger stress overshoot with a higher value of steady-state stress. The latter implies incomplete structural breakdown under the applied shear rate. This is an indication of even stronger three-dimensional interconnected microstructure due to the contributions of either CNT-CNT and/or CNT-BCP domains interactions.

Contrary to CNT, the CNT-PS filled nanocomposite sample exhibits a lower stress overshoot but similar steady-state stress in comparison to neat BCP. As indicated in the discussion of the frequency sweep experiment, the CNTs in nanocomposites containing $0.75 \mathrm{wt} \%$ CNT-PS cannot form a three-dimensional network. In other words, in the CNT-PS filled sample nanoparticles are uniformly dispersed in the BCP matrix. Because CNT-PS are well dispersed in the polymer matrix and do not form aggregates, no nanoparticle structural breakdown, caused by de-agglomeration of CNTs, is expected to occur during the applied shearing field, resulting in a similar steady-state stress than the neat BCP. Moreover, due to the orientation of neighboring PS domains along modified CNT-PS particles and consequent weakening of the strength of three-dimensional BCP domain microstructure, the nanocomposite sample displays a lower stress overshoot.

Time sweep experiments after the cessation of flow were utilized to provide more insight into understanding the effect of nanoparticle modification on BCP nanocomposite reorientation and restructuring. Figure $6 \mathrm{~b}$ depicts the recovery of storage modulus as a function of annealing time for neat $\mathrm{BCP}$ and the nanocomposite samples. It can be observed that neat $\mathrm{BCP}$ shows recovery behavior with slow rate at initial annealing time, followed by a higher rate at longer times. Moreover, the presence of nanoparticles results in slower restructuring kinetics. This influence is more prominent with modified CNT-PS. The main mechanism in BCP recovery is the reorientation of domains due to the Brownian motion, followed by three-dimensional microstructure formation between the randomized domains.

The higher initial value of storage modulus in CNT filled nanocomposite sample compared to neat $\mathrm{BCP}$ is attributed to the three-dimensional physical network structure between CNTs, 
which is not completely destroyed under the applied shear rate. On the other hand, the values of storage modulus for modified CNT-PS filled nanocomposite, which are similar to the neat $\mathrm{BCP}$, are related to the absence of any initial nanoparticle network structure.

Furthermore, the slower kinetic of restructuring in the CNT filled nanocomposite sample is an indication of strong interfacial interaction between CNTs and the neighboring domains, which leads to a longer recovery time. The decreased rate of recovery in the presence of CNT-PS is mainly due to the significantly increased interfacial interactions between the CNT-PS and PS domains, causing severely restricted PS segments with strong hindered reorientation motion.

Fig. 6

\subsection{Dynamic Mechanical Thermal Analysis}

DMTA analysis is one of the relaxation methods used to determine the structure and phase transformation of a material. This approach relies on measuring storage modulus ( $\left.E^{\prime}\right)$, loss modulus $\left(E^{\prime \prime}\right)$ and mechanical damping factor $\left(\tan \delta=E^{\prime \prime} / E^{\prime}\right)$ as a function of temperature. The temperature corresponding to the damping factor peak is taken as the glass-transition temperature or indicative for a transition associated to any other molecular motion. Figure 7 exhibits the results of damping factor vs. temperature for neat $\mathrm{BCP}$ and the nanocomposite samples. The samples display three peaks at around $-30{ }^{\circ} \mathrm{C}, 40-50{ }^{\circ} \mathrm{C}$ and $100-120{ }^{\circ} \mathrm{C}$ which can be assigned to the glass transition of ethylene-butylene (EB) domains, interphase regions and PS domains. The interphase is a transitional region between EB and PS domains. According to Zhou et al., weak crystals of EB exist in soft EB domains and at the interphase region. At temperatures around $25-55{ }^{\circ} \mathrm{C}$, these weak crystals of EB melt, which causes the aromatic rings of the PS block in the interphase region to move. ${ }^{[36]}$

Incorporation of CNT-PS into the BCP material leads to an increase in the area of $\tan \delta$ of rubbery EB domains, accompanied by a decrease of the corresponding area of the interphase 
region and the PS domains. These results reveal that the presence of CNTs promotes the microphase separation process because of the favorable interaction between CNT and PS segments. ${ }^{[37]}$ Microphase separation enhancement occurs by pulling out the EB and PS segments from an interphase region, allowing them to arrange in the corresponding neighboring domains. This phenomenon leads to the increase in the area of $\tan \delta$ of both distinct domains. As a consequence, the area of $\tan \delta$ of the interphase region decreased.

The greater thermodynamic affinity between CNT-PS and PS segments of the block copolymer in comparison to pristine CNT significantly promoted the microphase separation process, as implied by the considerably lower $\tan \delta$ area of the interphase region and the higher $\tan \delta$ area of EB domains. However, due to the arrangement of PS segments in the formed domains and considerable free volume reduction, segmental movement is restricted. Therefore, the damping associated to BCP PS domains decreased in the presence of modified CNT-PS in comparison to composite materials that contained pristine CNTs.

Fig. 7

In order to investigate the effect of thermal annealing on the microstructural development, the materials were placed in a vacuum oven at $130{ }^{\circ} \mathrm{C}$ overnight. Figure 8 depicts the temperature dependency of the damping factor tan $\delta$ for the samples after annealing. It can be observed that annealing influenced the BCP microstructure, which is clearly evident by changes in all three damping peaks. The area of $\tan \delta$ of rubbery EB domains increased while the area of peaks that correspond to the interphase region and the PS domains decreased. Moreover, the peak of the interphase region shifted to higher temperature. In our study, the samples were cooled to room temperature immediately after melt compounding at $220^{\circ} \mathrm{C}$. Thus, their microstructure is far from the BCP equilibrium morphology. Thermal annealing is known as an efficient approach to push the morphology towards the thermodynamically favored one. Thermal annealing imparts mobility to the system by enhancing the segmental entropy, thus enabling the BCP to 
attain its equilibrium structure. Following this mechanism, microphase separation progressed and interphase regions diminished.

The DMTA results of annealed samples allow one to conclude that it is unlikely for the system to reach its equilibrium morphology. Only a fraction of PS segments from the CNT-PS transfer into the PS domains, while the remaining PS segment units would be locally redistributed within the interphase region. Consequently, the mobility of PS segments in the interphase region and in PS domains would be hindered, leading to the observed shift of interphase peak position to higher temperature and also decreasing the area of $\tan \delta$ of interphase region and PS domains. Moreover, due to the higher number of EB segments in rubbery domains, the area of the rubbery damping peak increased.

It should be noted that the above mentioned microstructural changes, which occur in the interphase region, are less pronounced in the BCP nanocomposite samples, particularly in the presence of modified CNT-PS, confirming our previous conclusion that the presence of nanoparticles can assist the development of BCP microphase separation.

Although the peak position associated to PS domain in the in the BCP/CNT-PS composite shifted to higher temperatures, its area remained almost unchanged. This can be attributed to the high thermodynamic affinity between modified CNT-PS and PS segments. It slowed down the dynamics of BCP PS segments. As the consequence, these segments could not completely restructure within hard domains, causing a negligible change in the area of $\tan \delta$.

Fig. 8

\section{Conclusions}

In the present study, we have investigated the influence of CNTs and their surface modification on the microstructural evolution of nanocomposite samples based on a cylinder-forming SEBS 
triblock copolymer. PS brushes were grown onto the surface of CNTs by ATRP with the aim of improving the affinity of the nanotubes to the PS domains of the BCP. The pronounced lowfrequency solid-like behavior observed in linear oscillatory shear tests of CNT filled nanocomposite sample was an indication of 3D network structure formed between CNTs and/or between CNTs and the matrix. In contrast, for the nanocomposite sample containing modified CNT-PS no sign of nanotube induced 3D network could be observed, which was attributed to the improved interfacial interaction between modified CNT-PS and the BCP's PS segments. As evidenced by temperature sweep measurements, incorporation of CNT increases the microphase separation temperature, which could be associated with the confinement of BCP's chains between carbon nanotubes. On the contrary, the improved interaction of PS segments of the BCP with the PS chains tethered on the surface of modified CNT-PS restrained the motion of BCP chains and therefore their ability to form microdomains. This lead to a decrease in the BCP microphase separation temperature. The results of transient flow measurements supported the proposed effect of the CNT modification on the resulting self-assembled $\mathrm{BCP}$ microstructure. DMTA investigation revealed that nanoparticles enhanced the BCP segmental incompatibility, hence facilitating the development of microphase separation similar to thermal annealing. The results of the current study offer an insight into the effect of CNTs on the microstructural development of BCPs. This understanding can be used to prepare composite materials with tailor-made properties in a more rational fashion.

Acknowledgements: We acknowledge funding from the Swiss National Science Foundation (grant numbers PP00P2_159258, PP00P2_144697 and the NCCR Bio-Inspired Materials) as well as from the Swiss Government Excellence Scholarships (FCS). SEBS block copolymer was kindly provided by Kraton Performance Polymers. We acknowledge Marketa Ilcikova, Professor Jaroslav Mosnacek (Polymer Institute, Slovak Academy of Sciences) and Professor Danilo Carastan (Universidade Federal do ABC) for useful discussions. 
Keywords: SEBS block copolymer; CNT; ATRP; Structural development, Rheological characterization, DMTA

\section{References}

[1] M. Grzelczak, J. Vermant, E. M. Furst, L. M. Liz-Marzán, ACS Nano 2010, 4, 35913605 .

[2] F. S. Bates, G. H. Fredrickson, Phys. Today 1999, 52, 32-38.

[3] F. S. BATES, Science (80-. ). 1991, 251, 898-905.

[4] G. H. Fredrickson, F. S. Bates, Annu. Rev. Mater. Sci. 1996, 26, 501-550.

[5] B. H. Kim, J. Y. Kim, S. O. Kim, S. Y. Park, P. B. Messersmith, I. S. Choi, R. Langer, D. G. Anderson, H. Lee, S. O. Kim, et al., Soft Matter 2013, 9, 2780.

[6] Y. Mai, A. Eisenberg, R. J. M. Nolte, J. C. M. van Hest, S. P. Armes, A. J. Ryan, A. L. Lewis, G. Battaglia, S. Tan, A. Brisson, et al., Chem. Soc. Rev. 2012, 41, 5969.

[7] S. B. Darling, Prog. Polym. Sci. 2007, 32, 1152-1204.

[8] J. K. Kim, S. Y. Yang, Y. Lee, Y. Kim, Prog. Polym. Sci. 2010, 35, 1325-1349.

[9] D. J. Carastan, L. G. Amurin, A. F. Craievich, M. do C. Gon??alves, N. R. Demarquette, Polym. Int. 2014, 63, 184-194.

[10] D. J. Carastan, N. R. Demarquette, A. Vermogen, K. Masenelli-Varlot, Rheol. Acta 2008, 47, 521-536.

[11] Y. H. Ha, Y. Kwon, T. Breiner, E. P. Chan, T. Tzianetopoulou, R. E. Cohen, M. C. Boyce, E. L. Thomas, Macromolecules 2005, 38, 5170-5179.

[12] R. Krishnamoorti, A. S. Silva, C. A. Mitchell, J. Chem. Phys. 2001, 115, 7175-7181.

[13] A. Vazquez, M. López, E. Serrano, A. Valea, N. E. Zafeiropoulos, I. Mondragon, J. Appl. Polym. Sci. 2008, 110, 3624-3637.

[14] S. Choi, K. M. Lee, C. D. Han, Macromolecules 2004, 37, 7649-7662.

[15] M. Ilčíková, M. Mrlík, T. Sedláček, D. Chorvát, I. Krupa, M. Šlouf, K. Koynov, J. 
Mosnáček, Polymer (Guildf). 2014, 55, 211-218.

[16] G. Matzeu, A. Pucci, S. Savi, M. Romanelli, F. Di Francesco, Sensors Actuators, A Phys. 2012, 178, 94-99.

[17] H. J. Salavagione, S. Quiles-Díaz, P. Enrique-Jimenez, G. Martínez, F. Ania, A. Flores, M. A. Gómez-Fatou, Macromolecules 2016, 49, 4948-4956.

[18] I. Szleifer, R. Yerushalmi-Rozen, Polymer (Guildf). 2005, 46, 7803-7818.

[19] T. Hosseini-Sianaki, H. Nazockdast, B. Salehnia, E. Nazockdast, Polym. Eng. Sci. 2015, 55, 2163-2173.

[20] G. Mittal, V. Dhand, K. Y. Rhee, S.-J. Park, W. R. Lee, J. Ind. Eng. Chem. 2015, 21, $11-25$.

[21] G. Matzeu, A. Pucci, S. Savi, M. Romanelli, F. Di Francesco, Sensors Actuators A Phys. 2012, 178, 94-99.

[22] L. Peponi, L. Valentini, L. Torre, I. Mondragon, J. M. Kenny, Carbon N. Y. 2009, 47, $2474-2480$

[23] L. Peponi, A. Tercjak, J. Gutierrez, M. Cardinali, I. Mondragon, L. Valentini, J. M. Kenny, Carbon N. Y. 2010, 48, 2590-2595.

[24] N. Calisi, A. Giuliani, M. Alderighi, J. M. Schnorr, T. M. Swager, F. Di Francesco, A. Pucci, Eur. Polym. J. 2013, 49, 1471-1478.

[25] S. K. Yadav, S. S. Mahapatra, J. W. Cho, J. Y. Lee, J. Phys. Chem. C 2010, 114, $11395-11400$.

[26] H. Garate, M. L. Fascio, I. Mondragon, N. B. D’Accorso, S. Goyanes, Polymer (Guildf). 2011, 52, 2214-2220.

[27] M. Ilčíková, M. Mrlík, T. Sedláček, M. Doroshenko, K. Koynov, M. Danko, J. Mosnáček, Polymer (Guildf). 2015, 72, 368-377.

[28] M. Ilčíková, M. Mrlík, T. Sedláček, M. Šlouf, A. Zhigunov, K. Koynov, J. Mosnáček, ACS Macro Lett. 2014, 3, 999-1003. 
[29] J. D. Peterson, S. Vyazovkin, C. A. Wight, Macromol. Chem. Phys. 2001, 202, 775784.

[30] I. W. Hamley, V. Castelletto, Prog. Polym. Sci. 2004, 29, 909-948.

[31] L. G. Amurin, D. J. Carastan, N. R. Demarquette, J. Rheol. (N. Y. N. Y). 2016, 60, 175189.

[32] C. D. Han, Polymer Rheology, Oxford University Press, 2007.

[33] G. P. Kar, A. Bharati, P. Xavier, G. Madras, S. Bose, B. A. Wolf, Q. Tran-cong, T. Chang, I. C. Sanchez, Phys. Chem. Chem. Phys. 2015, 17, 868-877.

[34] S. Aoyama, Y. T. Park, T. Ougizawa, C. W. Macosko, Polymer (Guildf). 2014, 55, 2077-2085.

[35] S. Xin, Y. Li, H. Zhao, Y. Bian, W. Li, C. Han, Q. Dong, Z. Ning, L. Dong, J. Therm. Anal. Calorim. 2015, 122, 379-391.

[36] T. Zhou, A. Zhang, C. Zhao, H. Liang, Z. Wu, J. Xia, Macromolecules 2007, 40, 90099017.

[37] V. V. Ginzburg, Macromolecules 2005, 38, 2362-2367. 


\section{Figures}

Figure 1. Thermogravimetric analysis of neat CNT, CNT-OH, initiator-modified CNTs (CNT$\mathrm{Br}$ ) and CNT modified with polystyrene (CNT-PS). Analysis was performed under nitrogen atmosphere at a heating rate of $10^{\circ} \mathrm{C} \mathrm{min}^{-1}$.

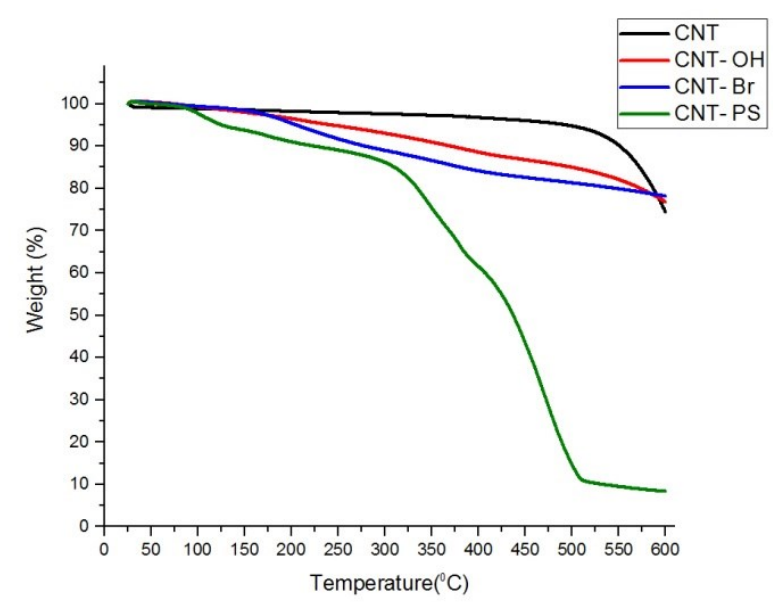


Figure 2. AFM pictures (phase mode) of annealed thin films of CNT/SEBS BCP nanocomposites. a) Neat $\mathrm{BCP}$ b) Nanocomposite of the $\mathrm{BCP}$ with unmodified CNTs. c) Nanocomposite of the BCP with modified CNTs-PS (The brighter and darker phase corresponded to the polystyrene and ethylene-butylene phases, respectively).
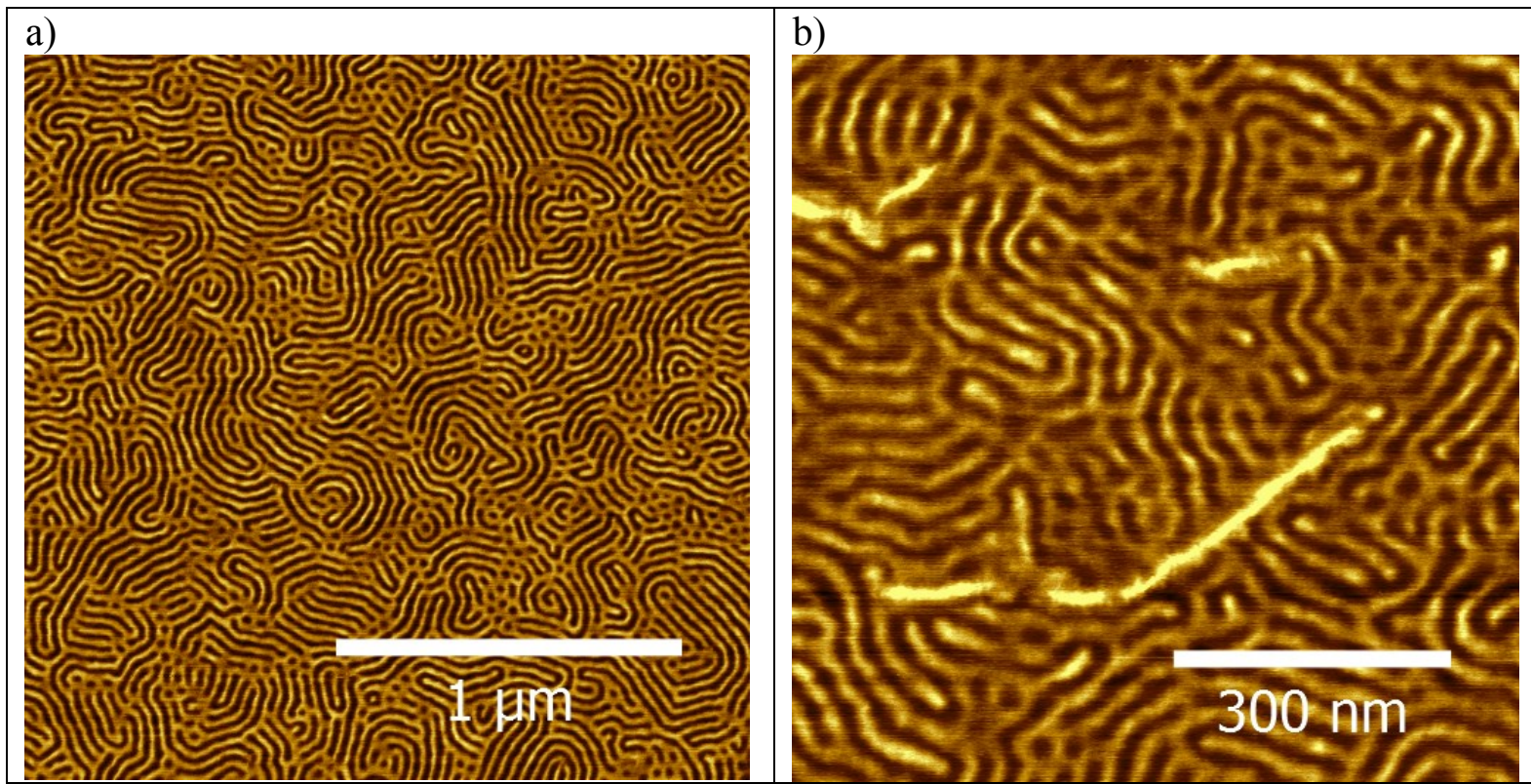

c)

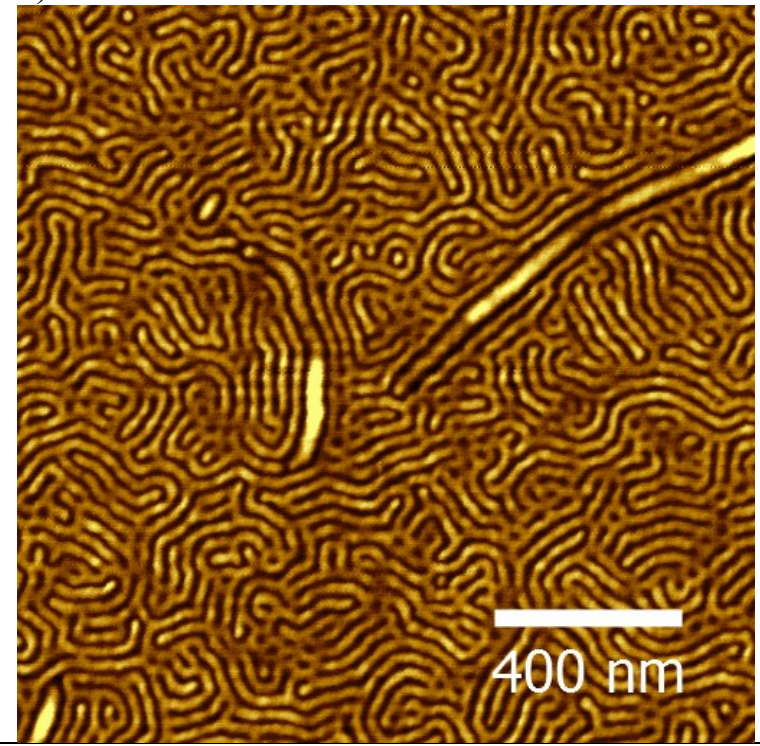


Figure 3. 1D SAXS plots for neat SEBS BCP (black curve) and nanocomposite samples containing $0.25 \mathrm{wt} \% \mathrm{CNT}$ (red) and $0.75 \mathrm{wt} \%$ modified CNT-PS (blue), recorded at room temperature.

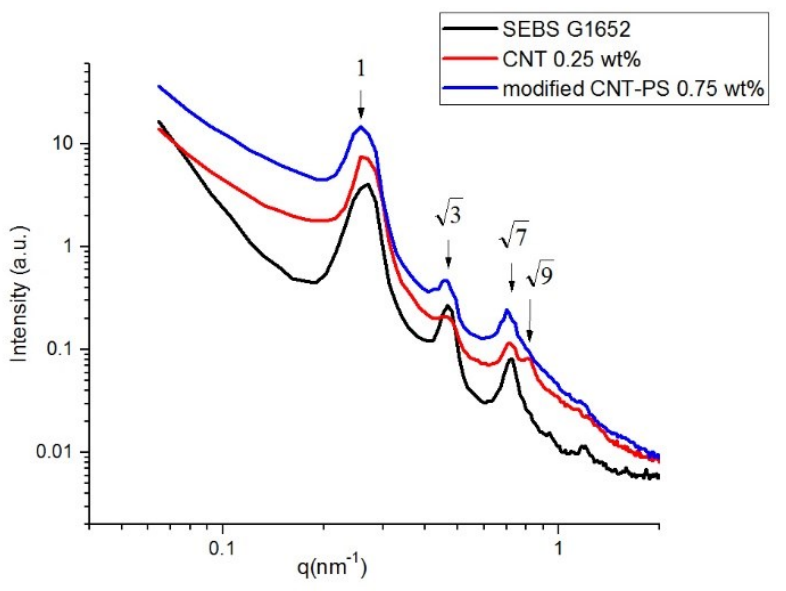


Figure 4. Storage modulus as a function of frequency for neat SEBS BCP and for nanocomposite samples containing pristine CNT and modified CNT-PS. All measurements were carried out at $220^{\circ} \mathrm{C}$.

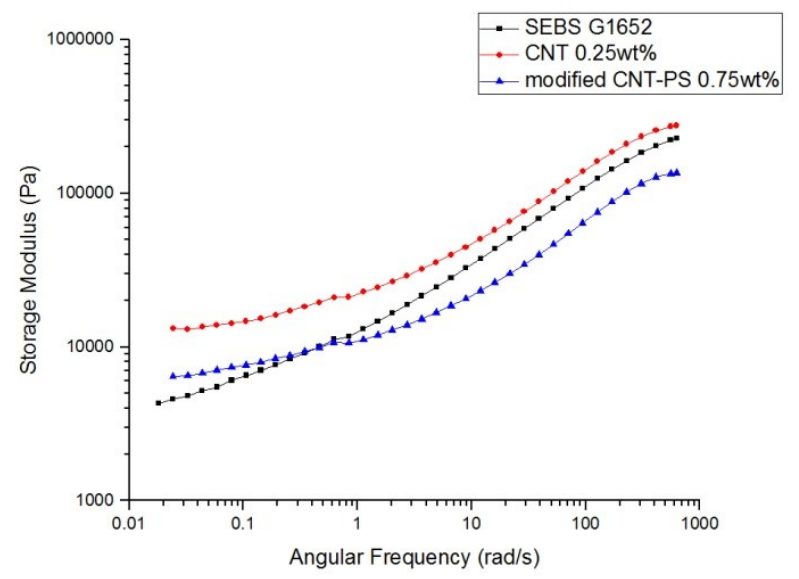


Figure 5. Storage modulus versus temperature for neat SEBS and nanocomposite samples containing pristine CNT and modified CNT-PS.

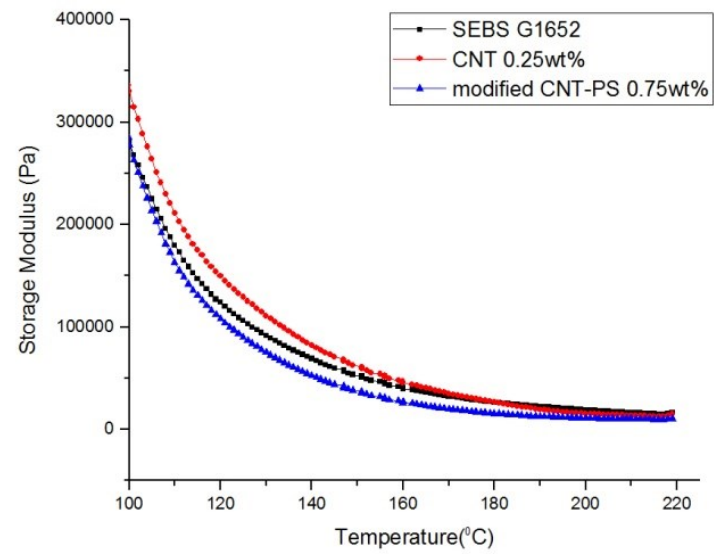


Figure 6. a) Stress as a function of time, b) Storage and Loss modulus recovery and c) normalized storage modulus after cessation of flow with shear rate of $1 \mathrm{~s}^{-1}$ for neat SEBS BCP and nanocomposite samples containing pristine CNT and modified CNT-PS at $\mathrm{T}=220{ }^{\circ} \mathrm{C}$.
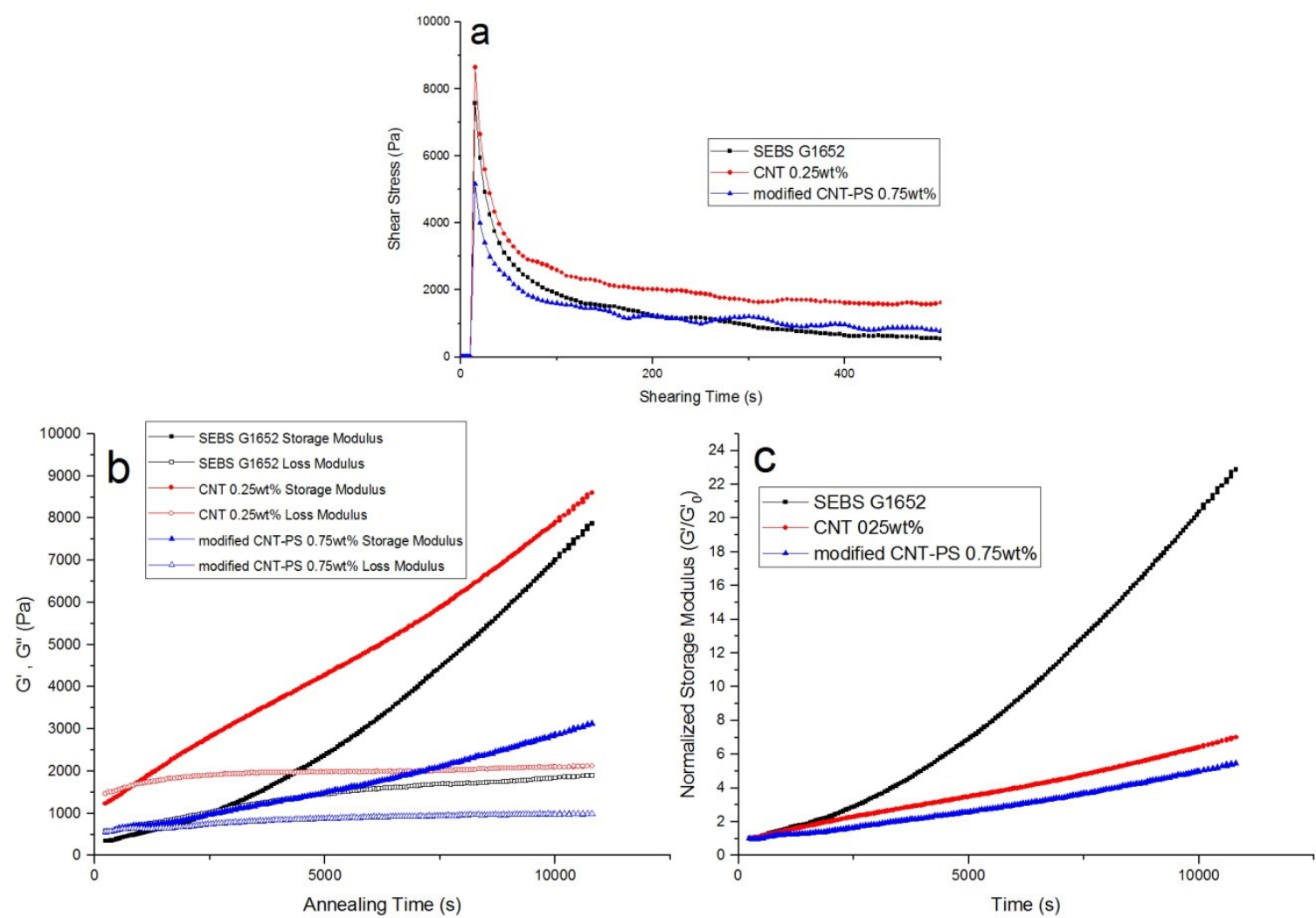
Figure 7. Damping factor ( $\tan \delta$ ) versus temperature for neat SEBS BCP and nanocomposite samples containing pristine CNT and modified CNT-PS.

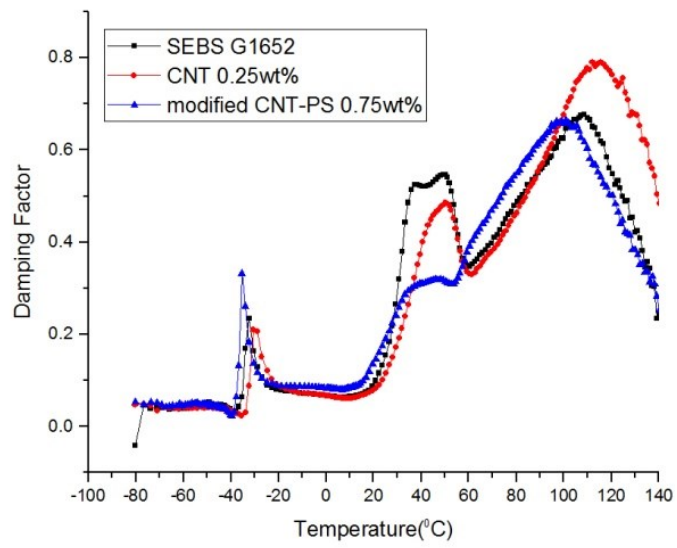


Figure 8. Damping factor ( $\tan \delta$ ) versus temperature for a) neat SEBS, nanocomposite samples containing b) $0.25 \mathrm{wt} \% \mathrm{CNT}$, and c) $0.75 \mathrm{wt} \%$ modified CNT-PS, before and after thermal annealing.
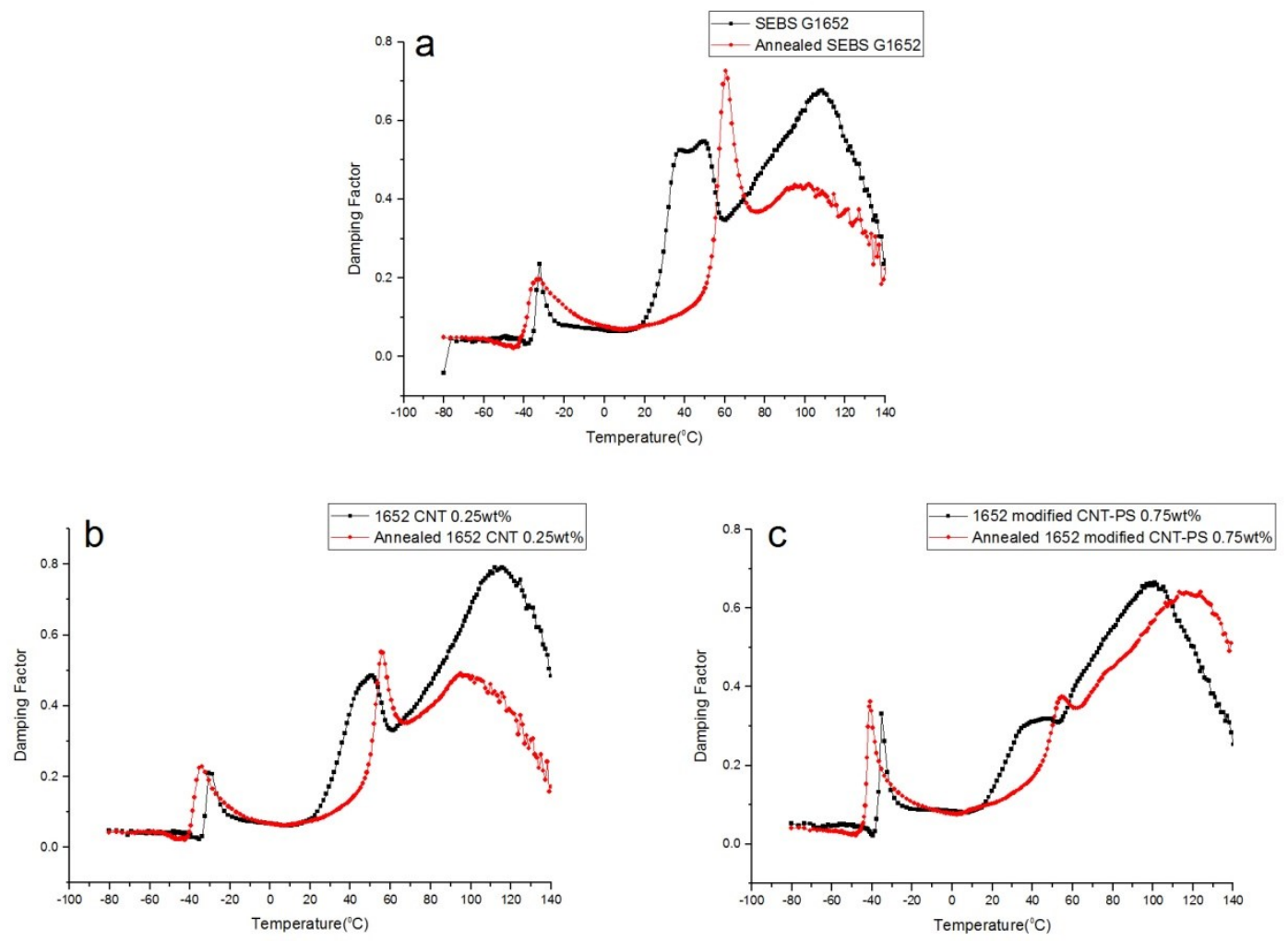
Block copolymer-based materials containing CNTs has drawn much attention in the literature. However, some aspects of these composite nanostructured materials, including the impact of CNTs and their modification on structural development have not been fully understood. This work aims at using modified CNTs with preferential interaction with one of the BCP's domains. Their influence on the structural evolution in comparison with pristine CNT has been studied.

Noushin Hasanabadi, Hossein Nazockdast*, Bernadetta Gajewska, Sandor Balog, Ilja Gunkel, Nico Bruns, Marco Lattuada*

Structural behavior of cylindrical polystyrene-b-poly(ethylene-butylene)-b-polystyrene (SEBS) triblock copolymer containing MWCNTs: On the influence of nanoparticle surface modification

ToC figure

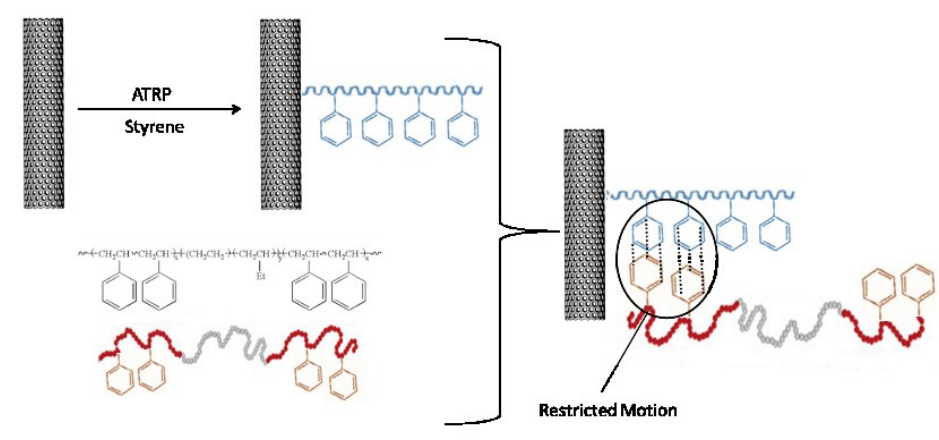

